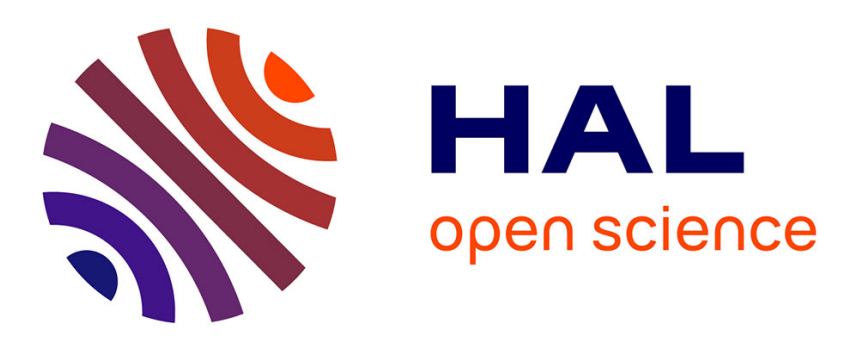

\title{
République, liberté et roman du Nouveau Monde dans la radicalité publiciste de Melvil-Bloncourt
}

Stéphane Douailler

\section{To cite this version:}

Stéphane Douailler. République, liberté et roman du Nouveau Monde dans la radicalité publiciste de Melvil-Bloncourt. Le Télémaque. Philosophie, Education, Société, 2021, La Commune de Paris ou la démopédie insurgée, 59, pp.95-112. 10.3917/tele.059.0095 . hal-03325768

\section{HAL Id: hal-03325768 \\ https://hal.science/hal-03325768}

Submitted on 29 Aug 2021

HAL is a multi-disciplinary open access archive for the deposit and dissemination of scientific research documents, whether they are published or not. The documents may come from teaching and research institutions in France or abroad, or from public or private research centers.
L'archive ouverte pluridisciplinaire HAL, est destinée au dépôt et à la diffusion de documents scientifiques de niveau recherche, publiés ou non, émanant des établissements d'enseignement et de recherche français ou étrangers, des laboratoires publics ou privés. 


\author{
République, liberté et roman du Nouveau Monde \\ dans la radicalité publiciste de Melvil-Bloncourt
}

Résumé: Pendant le mois d'avril 1871, au cours duquel le général Cluseret, délégué à la guerre de la Commune, travaille à la création d'une force armée organisée, il reçoit l'assistance, pour l'enrôlement de bataillons de marche et d'artillerie, d'une figure républicaine connue de la bohème littéraire du Second Empire, Melvil-Bloncourt. L'article examine comment celui qui fut un important rédacteur de la Revue du Monde Colonial, un député de Guadeloupe, un condamné à mort par contumace, un exilé à Genève et un intellectuel mulâtre odieusement caricaturé par A. Daudet dans Jack, réorienta au fil du temps sa compréhension des idées de république et de liberté en ajoutant, à la séquence qui place la Commune dans la suite de 1848, l'enjeu des guerres qui abolissent l'esclavage à échelle mondiale et redéfinissent ce qui mérite d'être appelé barbarie.

Mots clés: Melvil-Bloncourt, République de 1848, Commune de Paris, monde colonial, esclavage, barbarie, Jack, Salammbô.

Il existe une manière de désirer la liberté qui paraît pouvoir se soutenir tout particulièrement d'une volonté d'existence ou d'avènement d'un régime républicain. Hors de cette représentation, se laisse certes constater un riche et dense réseau d'innombrables libertés particulières qui savent se déployer sous d'autres régimes et qui peuvent être créditées d'extensions diverses au sein de formes de vie concédées, disputées, contractualisées. La république aurait cependant pour elle d'offrir un régime dans lequel les libertés particulières dériveraient cette fois d'une liberté que l'on désignerait mieux en la nommant «liberté en tant que telle». C'est celle-là qu'il conviendrait d'associer à la république et de le faire d'une façon si directe et si nécessaire que le sort même de cette dernière, c'est-à-dire la possibilité qu'elle soit ou ne soit pas, s'y trouverait engagé. Là où une telle représentation pénètre la trame des événements, elle tend à conférer à l'histoire de la république le trait d'être une séquence à éclipses. Dans les moments où, ainsi qu'il arrive, les libertés particulières sont restreintes, supprimées, confisquées au point de paraître faire s'évanouir la liberté en tant que telle, qui est espérée du régime républicain, ce serait alors la république elle-même qui verrait son existence comme mise en suspens pour un temps. C'est ce qui dans l'histoire française se serait passé, selon une représentation courante, lorsque la révolution de février 1848 dut céder la place à la sanglante répression menée par le général Cavaignac pendant les journées de juin 
suivies du coup d'État préparatoire à un second empire, puis lorsque, prenant le relais de ce dernier, la Troisième République se dévoila réfractaire à l'expérience de la Commune de Paris et y mit fin par un nouveau massacre au cours de la terrible semaine du 21 au 28 mai 1870, ainsi que par une action judiciaire impitoyable de condamnations à mort, à la prison, aux travaux forcés et à la déportation. Au long de ces événements, et dans un registre qui se sépara radicalement des hésitations et discussions politiques pour lesquelles un retour traditionaliste ou constitutionnalisé à la monarchie demeurait une option, une des façons de penser un mode de persévérance de la république consista alors certainement à nouer cette dernière plus étroitement que jamais, au sein d'une même et unique séquence, avec les avatars que connaissaient de leurs côtés les libertés. Comme si une sorte de fusion naturelle entre la république et une certaine liberté en tant que telle réglait pour l'une autant que pour l'autre les temps de leurs apparitions et de leurs évanouissements. Comme si c'était par et dans l'état de naturalité et d'évidence d'une même condition qu'elles se rejoignaient. À la manière pourrait-on dire - et c'est de cette expérience dont on se propose ici de tirer quelques fils - dont l'accès à la liberté, qui s'expérimente au long des processus d'émancipation hors de l'esclavage et de la servitude, a voulu et a su se donner une sorte de traduction irrécusable dans une représentation de la république au sein de laquelle, par nature en quelque sorte, n'existeraient, n'existeront, que des êtres pleinement assurés de leur condition de sujet libre.

\section{Aux jours de la libre parole}

On sait que de cette intrication intime entre l'idée de république et celle de liberté, Edgar Quinet fut une voix et une figure tant douloureuse qu'intransigeante ${ }^{1}$. Lors de l'exil auquel il se résolut à l'avènement du Second Empire et qui le conduisit pendant de longues années en Belgique et en Suisse, il reçut le 5 février 1861 une lettre signée (Suzanne) Melvil-Bloncourt où il put lire:

Monsieur et cher maître, je ne veux pas laisser partir mon camarade Chassin sans lui remettre pour vous un mot d'humble salut et de profonde reconnaissance. Sans que vous [vous en] soyez jamais douté, l'enseignement que vous m'avez donné pendant trois ans (de 43 à 46) au Collège de France m'a sauvegardé comme un talisman contre la pestilence universelle de ce temps maudit. J'étais un de ces ardents jeunes gens qui aux jours de la libre parole applaudissaient la vôtre ${ }^{2}$.

La lettre continue en signalant que son auteur, probablement inconnu d'Edgar Quinet, a écrit un article dans Le Progrès, journal de Port-au-Prince fondé par Exilien

1. Se reporter spécialement à G. Navet, «Exil et mémoire. Edgar Quinet», Hermès, nº 10, 1992/1, p. 225-232.

2. W. Alante-Lima, Melvil-Bloncourt. Le communard marie-galantais?, Saint-Maur-des-Fossés, Sépia, 2014, p. 32. 
Heurtelou, afin que «les Haïtiens connaissent le nom d'Edgar Quinet, apprennent à le vénérer comme celui d'un des hommes les plus grands et les plus honorables de notre époque». Les derniers mots de la missive, après qu'a été évoquée la mémoire d'un autre ancien auditeur de Quinet aujourd'hui enterré en Guadeloupe, sont pour affirmer que «notre race est peut-être sur le globe celle qui aime le plus la Révolution» et pour souhaiter ardemment le retour d'exil de Quinet:

Ce jour, oh! qu'il est long à venir [où] nous crierons de nouveau sous les vieilles voûtes de la Sorbonne comme au lendemain de février: Vive la République! ... Vous souvenezvous de ce jour-là ? Eh bien ce jour-là vous avez été sublime ${ }^{3} \ldots$

Cette confiance en une république liée à la liberté de la manière la plus intime, Suzanne Melvil-Bloncourt la maintient inentamée dix ans plus tard alors qu'il va se jeter dans «la fournaise de la Commune ${ }^{4}$. Commentant dans un courrier de février 1871 la capitulation des membres du Gouvernement de la défense, il écrit que «chacun d'eux mérite, avec justice, dix balles dans la tête». Et il ajoute:

Dans le gouffre de honte, de deuil, de trahison et d'ineptie [...] où se débat la France en lambeaux et toute ensanglantée, flotte, comme le drapeau de l'espérance, la confiance en la durée de la République. Aussi, que nos éternels ennemis n'entonnent pas encore un chant de triomphe sur le cadavre de la République; elle leur prouvera, avant longtemps peut-être, qu'elle est encore pleine de vie ${ }^{5}$.

Ce qui fait persévérer la république dans la vie à travers les séquences à éclipses qu'elle endure se donne en tout premier lieu, pour l'auteur de ces mots, dans la résonance persistante de ce qu'il appela dans sa lettre à E. Quinet les «jours de libre parole». Ce n'est pas seulement la république qui donnerait la liberté de parler, c'est tout autant la liberté de parler qui donnerait existence à la république pendant le temps de ses éclipses, y compris quand c'est une république, la Troisième République française à son aurore, qui ne cesse de démentir son propre nom. C'est dans la libre parole que république et liberté toucheraient à leur plus profond point de fusion. La défense, l'exercice, la propagation de cette fusion occupèrent en conséquence le plus clair des tâches que Melvil-Bloncourt s'assigna dans une infatigable activité de publiciste menée pendant plus de quarante années. Sa famille l'avait expédié en 1841 à Paris pour qu'il y poursuive ses études au lycée Louis-le-

3. Ibid., p. 33. Des ouvrages de souvenirs, celui par exemple rédigé par C.L. Chassin, avaient rappelé une protestation des étudiants de la Sorbonne le 20 mars 1851 contre la suspension du cours de Michelet au Collège de France à la suite de laquelle, une lettre à J. Michelet ayant été rédigée, une délégation étudiante décida d'aller la porter au domicile d'E. Quinet boulevard Montparnasse et en fut empêchée par des arrestations opérées par les forces de police. Melvil-Bloncourt est décrit dans ces pages comme ayant été «l'un des plus ardents excitateurs» de la manifestation, comme un aîné plus averti de la conduite à tenir face à la police et à la justice, et comme un lecteur passionné de l'œuvre d'E. Quinet. Voir Félicien. Souvenirs d'un étudiant de 48, Paris, E. Cornély et C ${ }^{\mathrm{ie}}, 1904$, p. 209 sq.

4. W. Alante-Lima, Melvil-Bloncourt..., p. 112.

5. Ibid., p. 113. 
Grand puis entreprenne, quatre ans plus tard, des études de droit ${ }^{6}$. Avait-elle songé à l'envoyer vers des «jours de libre parole» ? En un sens, certainement. Une légende familiale transgénérationnelle permet en effet de tisser un fil entre Suzanne MelvilBloncourt et ses descendants et neveux: Élie Bloncourt qui sera résistant et député SFIO; Tony Bloncourt, qui sera résistant, communiste et fusillé en 1942 au Mont Valérien; Gérald Bloncourt, qui, après les journées révolutionnaires en 1946 des Cinq Glorieuses où se joua le sort du gouvernement Lescot ${ }^{7}$, sera condamné à mort en Haïti et contraint de chercher un refuge en Martinique puis en France, où il fera une carrière de reporter-photographe au journal L'Humanité. Au départ de cette séquence, Suzanne Melvil-Bloncourt s'intègre pour sa part dans les groupes d'étudiants qui s'immiscent en 1848 dans tous les événements de la révolution de février. C'est là qu'il fait une expérience concrète des liens entre libre parole et république, qui, l'ouvrant à une compréhension initiale du chemin qu'il empruntera, lui fait apercevoir une continuité discursive menant des exercices appris sur les bancs de l'école vers les improvisations hasardées dans les clubs et réunions publiques, les harangues politiques tenues lors des journées décisives des révolutions, les articles de presse qui informent et instruisent les lecteurs, les professions de foi présentées aux électeurs. Dès 1846, il crée une Conférence Montesquieu conçue sur le modèle de ces groupes de travail étudiants dans lesquels, moyennant l'installation bricolée de quelque tribune, les «jeunes gens qui se préparaient à faire de la politique et, plus largement, à tenir un rôle sur la scène publique ${ }^{8}$ jouaient à discourir. Dans le même temps, il fonde un Journal des écoles qui veut accueillir les idées avancées et dans lequel, en 1847, on semble pouvoir reconnaître comme étant l'un de ses articles un texte sur l'abolition de l'esclavage, à l'instar d'un texte sur le même sujet qui paraît, quant à lui, dans La lanterne du Quartier Latin ${ }^{9}$. On le retrouve peu après, en mars 1848, commissaire à un Banquet des écoles présidé par Lamartine et Ledru-Rollin, avant qu'il ne se fasse remarquer encore et toujours comme fondateur d'un Club central des écoles situé place de la Sorbonne. Cette succession d'engagements aimantés par l'idée de république et centrés sur une parole qui saurait mêler performance oratoire et efficacité politique l'amène alors à concevoir un projet de plus grande ampleur. Avec deux autres contributeurs, il s'engage dans la réalisation d'un ouvrage intitulé La France parlementaire, que ses auteurs destinent à recueillir les « discours et rapports prononcés par les principaux orateurs des Assemblées depuis 1789 » afin - expliquent-ils - de «former l'éducation politique dans le temps

6. Voir G. Vapereau, Dictionnaire des contemporains, Paris, Hachette, 1880 et Le Maitron. Dictionnaire biographique du mouvement ouvrier et du mouvement social: https://maitron.fr/ spip.php?article65966, notice MELVIL-BLONCOURT Suzanne (vicomte), version mise en ligne le 26 juillet 2009, dernière modification le 4 juillet 2020. Pour toutes les références, se reporter aussi à W. Alante-Lima, Melvil-Bloncourt...

7. Se reporter à G. Bloncourt et M. Löwy, Messagers de la tempête. André Breton et la révolution de janvier 1946 en Haïti, Montreuil, Le Temps des cerises, 2007.

8. A. Martin-Frugier, «La formation des élites: les “conférences" sous la Restauration et la Monarchie de Juillet», Revue d'histoire moderne et contemporaine, t. 36, nº 2, avril-juin 1989, p. 213.

9. W. Alante-Lima, Melvil-Bloncourt..., p. 21. 
où nous vivons et édifier l'opinion sur les grandes opinions dont notre génération est préoccupée, la grande lutte parlementaire qui a présidé aux institutions qui nous régissent; cette lutte a été laissée dans l'ombre; de là une immense lacune dans l'histoire ${ }^{10}$. Cette "lacune», comme la suite le montrera, pourra s'entendre et se laisser combler de façons multiples.

Pour le moment, le coup d'État du 2 décembre 1851 interrompt l'ambitieuse publication. Pour ce travail comme pour l'esprit qui l'anime, plusieurs jours d'emprisonnement sont infligés à son auteur. D'autres incarcérations suivent rapidement quand Melvil-Bloncourt s'associe aux protestations et manifestations contre la suspension des cours de Mickiewicz et de Michelet au Collège de France, et se fait prendre dans plusieurs rafles. Mais la confiance républicaine qu'il place dans les beaux jours de la libre parole et dans la sédimentation active qui devrait en être confiée à des revues, recueils et ouvrages destinés au public l'accompagnera jusque dans sa dernière période politique, où, élu en 1871 par ses compatriotes aux fonctions de député de la Guadeloupe à l'Assemblée nationale et maintenu dans ce mandat par-delà la Commune jusqu'au 27 février 1874, il se souciera particulièrement de doter les communes de son pays natal de bibliothèques, de collections de livres, sans oublier de les compléter avec diverses copies de tableaux de peintres choisies dans la perspective de créer un musée ${ }^{11}$.

\section{Le métier d'écrire}

Aimer et unir au sein d'un même destin la république dans la liberté et la liberté dans la république est une chose. Postuler que c'est travailler à ce destin uni et prendre part à l'avènement de ses promesses que de porter les mots qui les disent dans des réunions publiques, des journaux et des revues, ou que d'en apprendre et d'en ciseler d'autres dans le retrait de l'étude en des temps moins favorables, est une autre chose. Celle-là implique que le point de fusion désiré entre liberté et république se maintienne haut et fort ici tout autant qu'à voix basse là-bas, qu'il se renouvelle, se déplace, se ressaisisse, se réoriente tout au long de l'exercice d'un verbe à visée publique qui traverse tout de même, au fil des ans, un nombre impressionnant de tribunes et de collaborations: contributions à La Vraie République

\section{Ibid.}

11. Ibid., p. 71-76. Il écrit à M. Léger, président du Conseil général: «Le choix des tableaux à reproduire m'ayant été laissé, [...] je choisis d'abord, comme un hommage filial à la Guadeloupe, le chef-d'œuvre du grand peintre Lethière: "Brutus condamnant ses fils". Je fis encore choix de deux autres chefs-d'œuvre» (O.D. Lara, La Guadeloupe physique, économique, agricole, commerciale, financière, politique et sociale. De la découverte à nos jours (1492-1900), Paris, Nouvelle librairie universelle, 1922, p. 272). Lethière était né en Guadeloupe d'une esclave affranchie. Il peignit à Paris des sujets antiques rivalisant avec David tout en leur donnant un tour crûment révolutionnaire et une touche préromantique. Louis XVIII le priva en 1816 de son élection à l'Institut comme il avait privé peu de temps avant le Chevalier de Saint-Georges de la direction de l'Opéra. On aimerait imaginer qu'un autre tableau choisi par Melvil-Bloncourt fut celui du "Serment des ancêtres », hommage à l'insurrection haïtienne (Lethière, 1822). 
de Théophile Thoré, au journal Le Peuple et à La Voix du peuple de Pierre-Joseph Proudhon, article dans la Revue des Deux Mondes, notices de La France parlementaire, contributions régulières pendant tout le Second Empire à la Biographie générale de Didot, au Dictionnaire universel de Maurice Lachâtre, au Dictionnaire Larousse, au Dictionnaire des communes de la France de Joanne, à la Revue du Monde Colonial, au journal L'Illustration. L'anthologie d'A. Lara Contribution de la Guadeloupe à la pensée française, publiée en 1936 dans le cadre du tricentenaire du rattachement des Antilles à la France, ajoutera à la liste de ces organes de presse, dans la notice qu'elle consacrera à Melvil-Bloncourt, toute une série d'autres ${ }^{12}$. De ce point de vue, Melvil-Bloncourt n'aura pas manqué, comme on le voit, de parcourir le chemin qui s'était présenté à lui aux premiers jours de la révolution de février. Ce sera certainement fort de ce travail accompli qu'il lui sera donné de rejoindre le 27 mai $1871^{13}$, en qualité d'élu de la Guadeloupe et pour un peu moins de trois ans, l'Assemblée nationale avant que, rattrapé par sa participation aux événements de la Commune, il ne soit condamné à mort par contumace et contraint à prendre la route de l'exil à Genève, où, mettant la dernière main à des travaux sur Voltaire, il rédigera sur ce dernier un épais ouvrage dont l'édition comprendra six volumes publiés sous des pseudonymes différents ${ }^{14}$. D'un côté, donc, le parcours de Melvil-Bloncourt se présente comme celui d'une parole obstinément libre se vouant à accomplir la promesse républicaine de liberté. Mais il se réalise aussi, d'un autre côté, comme trajectoire concrétisant, comme le signale un article de G. Sarlat paru dans le Nouvelliste de la Guadeloupe, un choix fait au début du $X_{X}{ }^{\mathrm{e}}$ siècle par des familles de l'île d'envoyer leurs enfants parfaire leur instruction en France, justifiant alors La Guadeloupe physique... d'O.D. Lara, au moment de le citer, de le ranger plus prosaïquement aux côtés de Privat d'Anglemont et de Victor Cochinat parmi les «trois hommes de couleur [qui] faisaient le métier d'écrire sous le second Empire ${ }^{15}$. Sous ce deuxième aspect, la séquence à éclipses de la république que Melvil-Bloncourt aura traversée l'aura également et plus spécialement inscrit dans la mémoire de ses contemporains comme artisan tantôt visible tantôt obscur d'innombrables entreprises éditoriales, non sans lui faire connaître, dès lors que celles-là entraient au cours de ces années dans une dimension industrielle, ce que O.D. Lara, le notant de Privat d'Anglemont, nomme «l'exploitation ${ }^{16}$.

12. A. Lara, Contribution de la Guadeloupe à la pensée française. 1635-1935, Paris, J. Crès, 1936 : «Pendant tout le Second Empire, Melvil-Bloncourt collabora à un grand nombre de dictionnaires, encyclopédies, revues et journaux, notamment [...] au Dictionnaire de Commerce et de Navigation, à l'Encyclopédie Générale, au Courrier de Paris, au Journal des économistes, au Siècle, à la Revue politique, au Courrier des Deux-Mondes, au Réveil, au Citoyen, à la Vérité, à l'Orphéon, à La production littéraire, etc., etc.».

13. Journal Officiel de la République française, 27 mai 1871.

14. Voir W. Alante-Lima, Melvil-Bloncourt...; A. Robert E. Bourloton et G. Cougny, Dictionnaire des parlementaires français, Paris, Bourloton, 1891, p. 335-336.

15. O.D. Lara, La Guadeloupe physique..., p. 253.

16. Ibid. Les pages consacrées à S. Melvil-Bloncourt sur le site de Gérald Bloncourt (http://bloncourt2. over-blog.com) en dressent un portrait qui rappelle entre autres sa fréquentation des bureaux où continuait malgré l'Empire de se confectionner le dictionnaire de Maurice Lachâtre, et rend 
Il n'y a par ailleurs rien à apprendre à ce sujet du roman d'A. Daudet, Jack. Mœurs contemporaines, qui y représenta Melvil-Bloncourt sous les traits, reconnaissables et reconnus, d'un publiciste cupide et prétentieux à la tête d'un sordide pensionnat. Rien qui permit d'éclairer véritablement la voie générale, propre ou décalée frayée par Melvil-Bloncourt au sein des devenirs républicains dont l'époque bouillonna. Il est connu que pour toute une partie remarquée du roman ${ }^{17}$, dans laquelle son héros, le jeune Jack, traverse l'épreuve du terrible apprentissage de la vie ouvrière aux forges d'Indret, A. Daudet, qui puisa son intrigue dans le drame réel d'un jeune homme dénommé Raoul Dubief qu'il avait pu suivre durant plusieurs années, prit soin de recourir à des notes prises sur le vif dans les faubourgs à l'époque de la Commune, qu'il se documenta et qu'il alla jusqu'à se rendre sur les lieux ${ }^{18}$. Le premier sous-titre qu'il choisit pour le livre qui parut initialement en feuilleton fut, conformément à cette démarche, «Histoire d'un ouvrier». Mais on sait aussi que pour sa description du Paris de la Bohème sous le Second Empire, laquelle motivera pour finir l'adoption de l'autre sous-titre, "Mœurs contemporaines ", il se fia en revanche à son expérience directe, ses rancœurs intimes, ses ressentiments sociaux, ses préjugés de race. Dans la préface à une réédition du roman ${ }^{19}$, il écrivit à propos de ses sources:

Les râtés et leur milieu m’ont coûté beaucoup moins de peine et de recherches. Moronval le mulâtre, a vécu lui aussi; il a collaboré à la Revue Coloniale et après 1870 fut quelque peu député. Il habitait, quand je l'ai connu, une petite maison à jardin aux Batignolles, et vivait d'une demi-douzaine de négrillons expédiés de Port-au-Prince ${ }^{20}$.

Dans la veine de la même facilité, le livre, en référence directe et transparente à Melvil-Bloncourt, prête aux membres de la Bohème qui honorent la pension Moronval de leurs rêves et travaux littéraires, la fondation, pour y recueillir leurs «inepties», d'une publication à laquelle A. Daudet choisit de donner pour titre La Revue des Races Futures ${ }^{21}$.

sensible que si l'assistance nombreuse et surveillée par la police qui vint à son enterrement le 9 novembre 1880 se quitta bien aux cris de «Vive la République!», elle semble en même temps avoir été particulièrement composée de représentants de journaux et divers organes de presse républicains.

17. L'école de (notre) république a fait aujourd'hui de l'ouvrage un classique des lectures scolaires.

18. Voir les deuxième et troisième parties d'A. Daudet, Jack. Moeurs contemporaines, Paris, E. Dentu, 1876. Se reporter notamment à C. Becker, «Alphonse Daudet: une vision ambiguë du monde du travail», Travailler, $\mathrm{n}^{\circ}$ 7, 2002/1, p. 53-62.

19. A. Daudet, Jack. Mours contemporaines, in Euvres complètes, t. II, Paris, E. Dentu, 1881 (édition précédée de l'histoire de ce livre).

20. Cité par W. Alante-Lima, Melvil-Bloncourt..., p. 169. La relecture de l'ouvrage proposée par W. Alante-Lima (p. 165-178) repère des traits empruntés à Melvil-Bloncourt chez plusieurs autres personnages du roman en dehors de Moronval, comme si l'existence réelle de Melvil-Bloncourt avait réussi à concentrer chez Daudet presque toute la hargne qu'il déverse dans le livre contre les «cénacles des ratés», et qu'il fait régulièrement prévaloir sur l'intérêt soulevé par le destin de Jack.

21. A. Daudet, Jack, II, 8. 
Une dédicace attend le lecteur à l'entrée de Jack: "Ce livre de pitié, de colère et d'ironie est dédié à Gustave Flaubert mon ami et mon maître. Alphonse Daudet ». Les mots, à n'en pas douter, sont pesés. En plus de l'hommage qu'ils rendent à Flaubert et de l'ambition qu'ils expriment de se placer dans son sillage, ils désignent par référence à la pitié, à la colère et à l'ironie un espace littéraire qu'A. Daudet fait le choix d'investir en présentant Jack comme élément inaugural de toute une série. Cet espace est visiblement aussi un espace politique d'écriture. Se saisissant des possibles littéraires de la pitié, de la colère et de l'ironie en même temps que de leurs mutations romanesques dont la prose flaubertienne donne l'exemple, A. Daudet proclame son intention d'écrire l'œuvre d'une réorganisation de mots, portraits, intrigues et points de vue capable de démêler et de ramener à une vérité vitale l'ensemble sensible, discursif, technique et moral des événements qui ont agité l'histoire récente. Dans la lignée du second sous-titre de son livre par lequel il élargit son propos aux "mœurs contemporaines", il reconnaît comme siens le labeur et l'ambition de se consacrer à une telle reprise et de se montrer à la hauteur de la tâche avec les moyens du métier d'écrivain auquel l'ancien secrétaire du duc de Morny et l'homme de lettres décoré en 1870 de la légion d'honneur des mains mêmes de l'impératrice Eugénie, qu'il est, est en train d'accéder. Tout en en excluant, du même geste, Melvil-Bloncourt et tous ses frères en écriture.

\section{Persévérance des mots, entêtement des faits}

Justice a évidemment été rendue depuis longtemps à la Bohème littéraire et républicaine du Second Empire à laquelle A. Daudet voulut dédaigneusement réduire le travail obstinément poursuivi par Melvil-Bloncourt. Les rêves, œuvres et impasses de cette Bohème font aujourd'hui intégralement partie de ce que nous continuons de chercher à comprendre de ce moment d'histoire et des orientations essentielles de notre propre situation. À ce sujet, aucun des traits prêtés dans Jack au « cénacle des ratés» de la "pension Moroval» n'a conservé la moindre chance de transmettre ni même d'approcher sur le mode de la caricature et de l'ironie quelque image digne d'être retenue de ceux parmi lesquels Melvil-Bloncourt évolua vraiment, et qui dans les faits furent, à côté de bien d'autres, Charles Baudelaire, Alfred Delvau, Henry Murger, Alexandre Privat d'Anglemont, Antonio Watripon, Étienne Carjat, Félix Nadar, Félix Pyat et Élisée Reclus. Considérer les processus et questionnements politiques qui traversèrent chacun d'entre eux nécessite définitivement des références tout autres. Mais il reste peut-être quelque chose à réfléchir et à saisir, non pas de la défectueuse caricature de Melvil-Bloncourt proposée par le personnage d'Évariste Moronval dans Jack, mais du geste de fiction romanesque qui le crée. Le manque d'empathie pour la générosité républicaine, le goût féroce de la déformation satirique, l'accommodement avec les puissants n'avaient en effet pas seulement tenu A. Daudet éloigné d'importants courants de son époque ni soustrait de ses centres d'intérêt toute réelle observation des manières variées de conjuguer liberté et république. Ils avaient aussi abrité une forme de haine, empoisonnée par 
des préjugés de race, de la concrète universalité de la république. Pour livrer à la caricature les revues philosophiques et littéraires, qui sous le régime de censure du Second Empire continuaient sourdement de travailler à la persévérance de l'idée de république en même temps qu'à leur survie propre, le roman d'A. Daudet met sous les yeux du lecteur une publication qui, s'occupant de mêler poésie, théâtre, politique éducative, science médicale et question sociale, ne reçoit nul autre nom que celui de Revue des Races Futures. Or cette forme haineuse d'ironie, dans laquelle A. Daudet élève à son paroxysme la distance qui le sépare désormais de la Bohème, touche, en dépit de son complet aveuglement à l'endroit des questions tant de la république que de la république universelle, à un point qui n'était pas sans être réellement en suspens pour ne pas dire mal engagé au sein des évolutions générales qui frayèrent la voie à une Troisième République stabilisée, et qui, entre autres, prirent la responsabilité de mener du complexe d'idées qui avait abouti le 27 avril 1848 à l'abolition définitive de l'esclavage en France et sur l'ensemble de ses territoires, vers celles qui entreprirent de colorier d'universalisme républicain une volonté conquérante propre à l'empire colonial français ${ }^{22}$.

C'est alors précisément une œuvre comme celle de Melvil-Bloncourt, qui, dans la diversité particulière de ses strates et dans l'engagement indéfectible de son inlassable labeur, permet d'observer quelques cheminements moins soumis au mouvement dominant et plus ouverts à l'avenir. Esquissons une succession de moments. Le premier d'entre eux est un essai qui ne dut pas manquer d'être remarqué en 1848 lorsqu'en collaboration avec son ami F. Servient ${ }^{23}$, Melvil-Bloncourt publie, dans un style polémique instruit des années de discussion de la question de l'esclavage, que le mouvement abolitionniste avait introduite dans le débat public, une diatribe intitulée «Réponse à l'article publié par M. Galos, ex-directeur des Colonies, sur l'émancipation ${ }^{24}$. La réponse se propose de faire entendre, à l'intention de la discussion ouverte par la toute récente promulgation de l'abolition, les effets proprement irrésistibles produits sur le débat public par la révolution de février et la proclamation de la république. L'heure a sonné de déchoir les savoirs de ceux qui furent anciennement envoyés dans les colonies pour les gouverner au nom de la monarchie, préserver les intérêts des colons et des armateurs, et différer le plus longtemps possible l'abolition de l'esclavage en rusant avec l'opinion. Il est temps maintenant de voir définitivement clair dans les recommandations politiques et économiques que les maîtres d'une époque révolue prétendent encore transmettre

22. Se reporter en particulier à M. Cottias, "Ces “hommes dangereux” de 1848. L'amnistie à l'épreuve de l'abolition de l'esclavage», Genèses, nº66, 2007/1, p. 30-50. Voir aussi, du même auteur, "Le silence de la Nation. Les "vieilles colonies" comme lieu de définition des dogmes républicains (1848-1850)», Outre-Mers. Revue d'histoire, nº 338-339, 2003, p. 21-45.

23. Jean-Pierre Ferdinand Servient, dit F. Servient. Voir Le Maitron, dictionnaire biographique, mouvement ouvrier, mouvement social: https://maitron.fr/spip.php?article178122, notice SERVIENT Jean Pierre Ferdinand dit SERVIENT F. par Pierre Baudrier, version mise en ligne le 23 janvier 2016, dernière modification le 4 juillet 2020 .

24. Revue des Deux Mondes, $1^{\text {er }}$ septembre 1848 et parue à part, éditée la même année par l'imprimerie Bonaventure et Ducessois. 
au nom de leur expérience. Lorsqu'ils préconisent de différer dans les colonies l'application à tous des droits de vote, de participation aux assemblées judiciaires et d'accès aux fonctions publiques, ils ne font rien d'autre que répugner à l'égalité inscrite dans la citoyenneté et tenter de sauvegarder des pouvoirs liberticides. Quand ils suggèrent de retarder l'introduction du salariat et des dispositions $\mathrm{du}$ code civil dans les contrats de louage et de contrer par le moyen de taxes le libre accès à l'industrie dans les villes ainsi qu'à la propriété dans les campagnes, ils continuent d'errer dans des législations différentielles destinées à rien d'autre en réalité qu'à aménager «la plaie honteuse de l'esclavage ${ }^{25}$ au sein d'une servitude ou d'un servage nouveaux, là où il conviendrait plutôt d'embrasser, à l'intention des peuples et territoires d'Outre-Mer, les voies du progrès et du bonheur. Publié six mois après la proclamation à Paris de la Deuxième République, quatre mois après l'abrogation de l'esclavage et à mi-parcours entre la sanglante répression des journées de juin 1848 et la promulgation de la nouvelle constitution, l'article de Servient et de Melvil-Bloncourt fait entièrement reposer l'état de liberté et d'affranchissement, dont les deux auteurs montrent qu'il se substitue à l'ancienne condition et en rebat toutes les cartes politiques et économiques, sur l'arrivée dans les colonies du fait républicain et avec lui de la république en tant que telle, c'est-à-dire de celle qui, "sous peine de mentir à son dogme, à son but, à elle-même» et « du seul fait de sa venue ${ }^{26}$, impliquerait que soit accomplie et tout aussitôt considérée comme déjà engagée l'œuvre de libération et de liberté. Pour préciser l'usage que l'article de Servient et Melvil-Bloncourt fait dans la situation des signifiants républicains, on semble pouvoir se reporter à une distinction, empruntée par A. Badiou à J. Lacan pour rendre justice à l'engagement poétique d'Aragon, entre un «objet-cause» dont on dira qu'il existe historiquement et qu'il soutient des expressions de désir, et une "cause-Idée» dont on dira qu'elle insère l'engagement qu'elle suscite de manière immédiate et absolue dans une finalité ${ }^{27}$. Rapportées à cette distinction, la république tout autant que la liberté en tant que telles, c'est-à-dire leur fusion la plus intime et la plus indéfectible, dont Servient et Melvil-Bloncourt entendent faire connaître que toute la discussion sur l'abolition de l'esclavage et sur ses conséquences serait passée sous sa règle, donnent à reconnaître la cause-Idée. Pour sa part, l'objet-cause, qui requiert qu'on le suive et l'identifie dans les vicissitudes de ses apparitions et disparitions, se manifeste au sein de cette même discussion dans la violente tension à laquelle Servient et Melvil-Bloncourt, dans la réponse qu'ils rédigent, soumettent les malheureux conseils qu'avait cru pouvoir dispenser l'exdirecteur des colonies et ex-ministre de la Marine du roi Louis-Philippe, démis par la République. L'existence réelle et vitale du désir de liberté, de république et de république universelle n'est pas ressaisie pour autant, comme M. Cottias le signale

25. F. Servient et S. Melvil-Bloncourt, «Réponse à l'article publié par M. Galos... », p. 3.

26. Ibid., p. 4-5.

27. A. Badiou, Radar poésie. Essai sur Aragon, Paris, Gallimard, 2020, p. 10. 
de manière générale pour ce moment d'histoire ${ }^{28}$, dans les troubles et espérances que l'attente de la république avait de facto suscités dans les colonies, et le désir de liberté n'est en réalité qu'ironiquement révélé sur un mode négatif qui retourne contre elle-même "l'élucubration ${ }^{29}$ de J.H. Galos. Les questions de la liberté du travail et le salariat, questions cruciales de la réinstauration de la République en 1848, sont ainsi évoquées dans des phrases moqueuses:

Selon lui [J.H. Galos], il eut fallu, pour maintenir le travail, accorder aux maîtres, durant dix années, la jouissance gratuite des labeurs de leurs nègres; et cela, apparemment, pour développer chez ceux-ci le goût du travail salarié. Pour notre économiste il n'est point, il parait, de stimulant plus efficace pour l'ouvrier que la certitude de ne tirer aucun profit de ses œuvres, et si l'on ne peut contraindre des hommes à travailler en leur promettant une juste rétribution, on est bien sûr de les y décider en leur assurant qu'ils n'ont rien à y gagner ${ }^{30}$.

Il se laisse remarquer aussi que la performance de ce persiflage, si on la considère dans sa teneur matérielle, est censée être accomplie tout entière par l'activité des publicistes. Ce n'est pas ailleurs que dans cette dernière, et dans une efficience qui lui est prêtée, que liberté et république indissolublement liées justifieraient de faire l'objet de prédications incessantes afin de rallier les lecteurs à leur cause, en même temps qu'elles fourniraient les occasions de déchiffrer des devenirs heureux et malheureux dans la trame des événements ${ }^{31}$.

Mais il s'avère alors aussi que le long parcours que Melvil-Bloncourt engage à partir de là dans les colonnes de ses prolifiques publications, sans se laisser distraire de la tâche en partie double d'œuvrer à la cause de la liberté et de la république, travaille insensiblement à l'éloigner du style satirique et autres figures rhétoriques calquées sur les exercices d'éloquence de ses débuts. Qu'il ait eu à persister dans le métier d'écrire au sein d'un système de prudences imposées par la censure du Second Empire ou qu'il ait eu à satisfaire des commandes de presse plus spécialisées, ses articles montrent un tour plus positif. L'orientation perce notamment en 1861 dans une nouvelle réfutation engagée avec vigueur contre une détestable brochure due cette fois à Achille Poussielgue, ex-attaché de l'ambassade de France à Washington, intitulée Homme ou singe ou la question de l'esclavage aux États-Unis ${ }^{32}$. Non seulement Melvil-Bloncourt pourfend de nouveau jusqu'à

28. Articles cités supra. Se reporter aussi à ses travaux plus récents tels que «Une Afrique globale au prisme de la mémoire de l'esclavage » dans l'Histoire générale de l'Afrique, à paraître aux éditions de l'UNESCO.

29. F. Servient et S. Melvil-Bloncourt, «Réponse à l’article publié par M. Galos... », p. 4.

30. Ibid., p. 9.

31. Voir A. Badiou, Radar poésie, p. 10-11.

32. A. Poussielgue, Homme ou singe ou la question de l'esclavage aux États-Unis, Paris, E. Dentu, 1861. L'auteur, qui se présente en adepte convaincu des «idées démocratiques» (p. 3), prétend vouloir protéger ces dernières de «l'exaltation du nègre prêchée par tant de grands esprits en Europe» (p. 3) et d'une confusion que des journaux auxquels il est abonné, le Siècle et l'Opinion nationale, feraient sans réfléchir en «mettant sur le même plan l'esclavage blanc et l'esclavage noir» (p. 3) lorsqu'ils dénoncent le servage russe ou polonais. Il oppose à ces idées un racisme 
l'ironie la plus cruelle l'ouvrage et ses tristes banalités à cent lieues de pouvoir éclairer le moindre lecteur, mais il choisit encore d'y ajouter, moins en direction de l'auteur que de la "moralité publique ${ }^{33}$, d'importants développements sur la question de l'esclavage aux États-Unis, c'est-à-dire sur le véritable objet qu'il saisit l'occasion de traiter ${ }^{34}$, pour lesquels il convoque l'histoire naturelle, la philosophie, les avis et témoignages des meilleurs auteurs et connaisseurs, la science historique, l'économie politique, «toutes choses dont [M. Poussielgue semble] ne pas même soupçonner l'existence ${ }^{35}$. Ce texte, avec d'autres de même ambition, n'atteste pas seulement un déplacement des intérêts de Melvil-Bloncourt vers un vaste spectre de questions qu'il va prendre en charge dans le cadre d'une collaboration de plusieurs années avec la Revue du Monde Colonial et dans d'autres contributions: économie politique des pays d'Amérique et de la Caraïbe, chronique de critique littéraire, chronique des événements d'Amérique du Nord, études d'histoire des républiques hispano-américaines, recueil d'actes d'héroïsme dans le monde colonial ${ }^{36}$. Il n'inaugure pas seulement, par la diffusion que Melvil-Bloncourt prend soin d'en faire aux États-Unis et dans les pays de la Caraïbe, une extension de son travail de publiciste à de nouveaux publics ${ }^{37}$. Mais il réoriente aussi l'espace de pensée et d'intervention dans lequel Melvil-Bloncourt continue de pister les succès de la liberté, l'acquisition que les peuples font de leurs droits et la visible croissance du mouvement d'abolition de l'esclavage, en accentuant un glissement de la fusion entre liberté et république en tant que telles entrevue en 1848 vers la cause-Idée d'une république universelle appelée à en prendre un relais inventif.

\section{Archéologie barbare}

Élargissant ses sujets d'intérêt et d'écriture, s'extrayant de la stricte guerre des discours, Melvil-Bloncourt paraît se tourner alors vers une vaste palette de questions agitées dans l'actualité. Prenant sa part, au sein de la Revue du Monde Colonial, de la communication d'événements concernant la Caraibe, il plaide ainsi la cause

virulent à l'endroit de la race noire, qu'il justifie au nom d'une hiérarchie descendante du vivant qui mènerait de l'homme à l'orang-outang et de là à la plus infime création dotée de vie. Cette hiérarchie conférerait à l'homme blanc «le droit de demander au nègre, qui est son inférieur par la volonté de Dieu, de lui rendre des services domestiques» (p. 4).

33. S. Melvil-Bloncourt, recension de Homme ou singe ou la question de l'esclavage aux États-Unis, Revue du Monde Colonial, organe des intérêts agricoles, industriels, commerciaux, maritimes, scientifiques \& littéraires des Deux-Mondes, $2^{\mathrm{e}}$ série, t. V, 1861, p. 144.

34. L'impression du titre de la recension de Melvil-Bloncourt inverse expressément la taille des caractères entre «Homme ou singe » et «La question de l'esclavage aux États-Unis » par rapport à la brochure d'A. Poussielgue.

35. S. Melvil-Bloncourt, recension de Homme ou singe..., p. 182.

36. Voir les livraisons de la Revue du Monde Colonial entre 1861 et 1865.

37. On trouve une importante contribution à ce sujet dans un article de B. LaPointe, " Moral electricity": Melvil-Bloncourt and the trans-Atlantic struggle for abolition and equal rights ", Slavery \& Abolition. A Journal of Slave and Post-Slave Studies, vol. 40, 2019, p. 543-562: https://www. tandfonline.com/eprint/Vd6nDt2TMhJkVfmthXx8/full. 
de la Guadeloupe dans une discussion avec la Martinique portant sur le choix des points d'escale des liaisons transatlantiques ${ }^{38}$. Peu après on le voit signaler, sur la base d'une information parue dans le journal de Guadeloupe Le Commercial, un fait d'armes, qui, ayant impliqué dans la guerre du Mexique un «valeureux petit corps de troupes insulaires", démontre à ses yeux la persistance du courage chez ceux qui, Guadeloupéens et Martiniquais, s'étaient jadis battus pour leur liberté contre les Anglais et les troupes napoléoniennes, mais qui l'incite aussi à ajouter ce commentaire:

Mais combien eussions-nous préféré voir leurs brillants coups d'essai dans la guerre se diriger contre les esclavagistes du Sud! Le Mexique, - sous la présidence du mulâtre Guerrero, lequel paya de sa glorieuse vie cet acte de suprême justice, - a été, qu'on s'en souvienne, la première terre qui, en Amérique, ait aboli l'esclavage ${ }^{39}$.

Tout est en place, dès lors, pour qu'en qualité de collaborateur d'une revue qui se dévoue à tous les intérêts de la France coloniale, qui possède des abonnés en Outre-Mer et qui compte parmi ses rédacteurs de nombreux anciens saintsimoniens et fouriéristes passés par Le Peuple de P.-J. Proudhon et le journal d'obédience libérale L'Algérie nouvelle de Clément Duvernois, Melvil-Bloncourt développe une trajectoire propre dans laquelle la libération de l'esclavage, les actes et pensées qui scandent sa réalisation historique, les batailles en cours, les problèmes qui surgissent dans le temps de l'affranchissement, les solidarités qui se nouent entre abolitionnistes prennent le pas sur toute autre question et marquent de leurs empreintes spécifiques l'engagement général en faveur de la république et de la cause de la liberté qu'il partage à des degrés divers avec la revue ${ }^{40}$. Citons un autre épisode. S'étant à partir de 1862 vu confier par A. Noirot une chronique bibliographique de critique littéraire ${ }^{41}$, et choisissant de rendre compte d'une parution en français des œuvres du poète né à Cuba d'un père de race noire et d'une mère européenne, Gabriel de la Conception (dit Placido), Melvil-Bloncourt lui consacre une notice qui le présente comme le «premier poëte des Antilles [...] et de la poésie hispano-américaine ${ }^{42}$. L'article raconte son enfance, ses ouvrages, ses idées démocratiques, sa fin tragique quand une obscure dénonciation le mène devant un peloton d'exécution. Puis Melvil-Bloncourt ajoute, au sujet des paroles

38. S. Melvil-Bloncourt, «Du choix définitif du point d'escale des Transatlantiques » et «Question des Transatlantiques», Revue du Monde Colonial, $2^{\mathrm{e}}$ série, 1862, t. VI, p. 400-415 et t. VII, p. 351-364. La Compagnie générale transatlantique faisait de la publicité dans les colonnes de la revue.

39. S. Melvil-Bloncourt, «La Guadeloupe et la Martinique au Mexique», Revue du Monde Colonial, $2^{\mathrm{e}}$ série, t. VII, 1862, p. 508.

40. Sur la fondation de la Revue du Monde Colonial et sa ligne politique éditoriale en matière de colonisation, se reporter en particulier à F. Manchuelle, "Origines républicaines de la politique d'expansion coloniale de Jules Ferry (1838-1865) ", Revue française d'histoire d'outre-mer, t. 75, $\mathrm{n}^{\circ} 279,1988$, p. 185-206.

41. Voir «Bibliographie», Revue du Monde Colonial, 2e série, t VII, 1862, p. 525.

42. S. Melvil-Bloncourt, «Critique littéraire», Revue du Monde Colonial, 2e série, t VIII, 1863, p. 366. 
de liberté que Placido avait adressées à ses compatriotes dans un pays sous le joug espagnol:

Mais ici je ne comprends pas le mulâtre Placido. De quoi se mêlait-il? A lui qui n'était pas citoyen dans son pays, que lui importait le vasselage ou l'indépendance de Cuba? Ne valait-il pas mieux avant tout, surtout, travailler par la pensée et l'action à la conquête de ses propres droits et aussi à la délivrance de ses frères les nègres? Du haut du môle Saint-Nicolas, les exemples glorieux de Chavannes et d'Ogé, ces illustres martyrs de Saint-Domingue, devaient resplendir à ses yeux, - il n'avait qu'à les imiter ${ }^{43}$.

Cette manière de ponctuer la question de la liberté, dans laquelle se laisse reconnaître l'œuvre obstinée d'une réorientation, traversera inlassablement les articles de Melvil-Bloncourt. Il manifeste dans ce cadre un intérêt toujours plus passionné pour la réalité des affrontements armés, depuis les heures glorieuses de la révolution haïtienne jusqu'à tous les événements de la guerre de Sécession qui se déroule au cours de ces années et dont il scrute les moindres détails grâce aux contacts qu'il a noués avec les éditeurs noirs de la revue bilingue New-Orleans Tribune ${ }^{44}$. Non seulement il salue le moindre fait d'armes mais il cherche visiblement aussi à prendre la mesure de ce que représente la présence, aux côtés des 750000 soldats de l'armée du général Grant, de "plus de 200,000 nègres [...], peut-être, la plus formidable agglomération de nègres armés qui ait été vue sur le globe ${ }^{45}$. Une sorte d'épopée se dessine sous ses yeux qui serait apte à élever à sa signification historique l' «horrible guerre de l'Union américaine» et ses "fleuves de sang» ${ }^{46}$. Telle bataille qui aux premiers jours de mai 1864 opposa pendant toute une semaine les Fédéraux commandés par le général Grant et les Confédérés conduits par le général Lee, et la victoire des premiers sur les seconds, lui inspirent, pour « rapporter ici les détails de cette lutte de géants", un récit épique et coloré de cinq pages ${ }^{47}$. À le suivre à travers les multiples notices, chroniques et correspondances qu'il confie à la Revue du Monde Colonial, on ne peut en réalité se défaire de l'impression qu'une sorte d'œuvre ou idée d'œuvre s'y esquisse. Comme si les interminables combats pour l'abolition de l'esclavage dans la réalité politique autant que dans les esprits, en attente encore de nombreuses victoires dans le temps où il écrit et où se joue sur cette question toute la construction des États des Amériques, exigeaient davantage que la réaffirmation d'une juste cause au gré des dépêches en provenance des lieux de conflits et des débats parlementaires, autre chose encore que la création fraternelle d'une souscription en faveur des esclaves affranchis des États-Unis «au fur et à mesure que la victoire les tirerait de la servitude ${ }^{48}$, autre chose même que l'exaltation des

\footnotetext{
43. S. Melvil-Bloncourt, «Critique littéraire», p. 369.

44. Cette collaboration est restituée avec précision dans l'article déjà cité de B. LaPointe.

45. S. Melvil-Bloncourt, "Chronique de l'Amérique du Nord», Revue du Monde Colonial, $2^{\mathrm{e}}$ série, t. XI, 1864, p. 93.

46. Ibid., p. 464.

47. Ibid. p. 457-461.

48. S. Melvil-Bloncourt, "Souscription en faveur des affranchis des États-Unis d'Amérique», Revue du Monde Colonial, t. XV, avril 1865, p. 5.
} 
héroïsmes passés et présents ou que la démonstration documentée des capacités et performances méconnues de peuples entiers. Comme si toute cette accumulation de petits actes et de données patiemment recueillies et livrées au public ne constituait encore qu'une sorte d' "archéologie» pour un plus ample travail.

Il est connu que c'est autour des significations du mot "archéologie» que s'était jouée en particulier la réception au même moment de Salammbô de Flaubert. Et parallèlement aux prétentieuses remarques que s'était cru autorisé à faire un archéologue conservateur des Antiques au musée du Louvre, et auxquelles un Flaubert assez exaspéré avait fini par répondre, Sainte-Beuve, dans une recension détaillée parue en trois livraisons dans Le Constitutionnel ${ }^{49}$, s'était en effet largement expliqué par l'archéologie, par le recours que Flaubert y aurait fait, l'étrangeté qu'il avait ressentie devant une œuvre d' " antiquaire [qui] s'éprenait d'une civilisation perdue, anéantie, et ne visait rien moins qu'à la ressusciter, à la recréer tout entière». Au nom de l'art, il y avait opposé un besoin, non satisfait par le livre, de «sympathie humaine». Il était prêt - écrivait-il - à entendre cette sympathie dans son acception la plus vaste, mais il n'imaginait pas pouvoir l'éprouver dans un roman ou poème "tout archéologique» relatant une "guerre perdue, enterrée dans les défilés ou les sables de l'Afrique», qui, à la différence par exemple du duel et de la guerre acharnée que s'étaient livrées Carthage et Rome, n'avait rien engagé qui concernât la civilisation future ou la nôtre. Salammbô est l'ouvrage par lequel Melvil-Bloncourt inaugure de son côté la chronique littéraire dont il prend la responsabilité au sein de la Revue $d u$ Monde Colonial ${ }^{50}$. On peut supposer, mais non pas véritablement savoir, qu'il a lu les critiques et les doutes exprimés par Sainte-Beuve dans Le Constitutionnel. Il en propose quoi qu'il en soit un tout autre mode de résolution. Sans doute questionne-t-il à son tour, dans le souvenir des leçons républicaines de Michelet et de Quinet enseignant à imiter l'Antique ${ }^{51}$, la part d'humanité partageable qui aurait réussi à subsister au sein du pari littéraire tenté par Flaubert dans Salammbô. Car à quoi et à qui accrocher sa sympathie quand l'auteur a inventé d'affliger l'un des héros d'une «lèpre pâle, étendue sur tout son corps, [qui] lui donnait l'apparence d'une chose inerte", et attribue à un autre (Hamilcar) le «caractère d'une sorte de Gobseck armé» au lieu d'admirer ses vertus et ses courages? Où sont, si on rapproche Salammbô de Sethos, roman écrit lui aussi sur Carthage plus d'un siècle auparavant par l'abbé Terrasson, les allusions politiques, les audaces sociales, la préoccupation de justice qui donnent aux ouvrages un but et une moralité? Des embarras, donc, des réserves qui sur divers points recoupent les plus malveillantes des recensions parues les jours précédents ${ }^{52}$, mais qui ne s'acheminent vers aucun rejet hostile. Melvil-Bloncourt signale ici un charmant dialogue, là une description faite avec une extrême délicatesse, ailleurs

49. Sainte-Beuve, «Salammbô par M. Gustave Flaubert», Le Constitutionnel, 8, 15 et 22 décembre 1862 .

50. S. Melvil-Bloncourt, «Critique littéraire», p. 68-76.

51. S. Melvil-Bloncourt, "Leconte de Lisle», Revue du Monde Colonial, $2^{\mathrm{e}}$ série, t. VI, 1862, p. 344.

52. Il cite celle de Théophile Silvestre, parue dans Le Figaro le 8 janvier 1863, qui est passée à la postérité comme exemple pur d'une méchanceté gratuite et aveugle à ce dont elle prétend parler. 
un passage saisissant de grandeur et d'éclat qui ferait penser à du Corneille et $\mathrm{du}$ Hugo harmonieusement fondus dans une prose savante. Un autre passage encore, dans lequel Flaubert décrit dans son horreur et dans son abomination le traitement que les Carthaginois réservaient aux esclaves, échappe à son tour, et sans doute de façon plus originale, au rejet:

Qu'on ne se récrie pas! Ici l'auteur est en pleine réalité vraie. Mais c'est l'esclavage moderne qu'il a dépeint. Partout où cette odieuse institution persiste, c'est encore ainsi que se passent les choses ${ }^{53}$.

C'est que même à supposer, avec Sainte-Beuve, que l'évocation archéologique d'un petit affrontement oublié entre Tunis et Carthage n'aurait su fournir à la Salammbô de Flaubert aucun autre sujet que celui d'une petite haine locale entre barbare et barbare, une haine qui ne nous ferait rien à «nous", il faut veiller à savoir qui l'on nomme barbare et se garder d'oublier les modernes dans la question. Melvil-Bloncourt a appris, au cours de ses années d'écriture et d'enquête dans la presse comme au long de la sourde polémique qu'il y mène, tissant un fil à la fois d'histoire et d'actualité, à discerner ce qui échappe à nombre de ses contemporains et à une grande partie des républicains qui l'entourent, à savoir qu'une imagination non corrigée par l' «archéologie» abrite et cache aussi bien, dans les imitations idéales de l'Antique qu'elle propose, des traits de barbarie moderne inaperçue. Rendre vie à Carthage, qui a laissé une profonde trace dans l'histoire mais sur laquelle d'innombrables bourreaux ont fait passer au fil des siècles comme un vent de malédiction, aurait eu un sens - écrit-il - si le projet avait été, par effet de la «sympathie qu'on doit au malheur», de "défendre enfin, armé de la Science et de l'Invention, la cité punique contre les calomnies de Tite-Live, les réticences de Polybe ${ }^{54}$. Mettre sous les yeux l'horreur inévitablement attachée à la guerre avec une précision toute chirurgicale instruite des champs de carnage modernes d'Eylau et de Wagram aurait pu se comprendre s'il ne s'était pas agi de pousser "un cri - inconscient je le crois - de l'homme du Nord contre l'homme du Midi » ${ }^{55}$ voire de jeter «après les Romains, après les Barbares, par-dessus deux mille ans, sa pierre de Gaulois à la patrie d'Annibal » ${ }^{56}$. Melvil-Bloncourt perçoit-il que «l'archéologie» de Salammbô pouvait être lue à la lumière des terribles journées de juin 1848 et de leur appropriation par Flaubert comme moment d'insurmontable crise, limite et défaite de la parole publique politique ${ }^{57}$ ? De manière «inconsciente» et plus

53. S. Melvil-Bloncourt, «Critique littéraire», p. 73.

54. Ibid., p. 70 .

55. Ibid., p. 76.

56. Ibid., p. 70 .

57. Dans sa lettre de réponse à Sainte-Beuve, Flaubert, qui énonce d'emblée qu' «il n'y a rien de plus compliqué qu'un Barbare», rappelle explicitement les "Mobiles en 48 » et «ce qui se passe actuellement aux États-Unis». Il argumente en disant qu'introduire dans son roman, comme Sainte-Beuve le lui avait suggéré, un philosophe, un «raisonneur chargé de nous faire un cours de morale ou commettant de bonnes actions, un monsieur enfin sentant comme nous » n'aurait produit qu'un contraste facile, « un contraste voulu et faux », et déclare qu'il « regarde les Barbares 
large, peut-être. En tout cas, renvoyant la possible solution des problèmes qu'il croit apercevoir vers ce que l'abbé Terrasson avait déjà tenté de son côté avec Sethos dans une forme certes bien inférieure, c'est-à-dire malgré tout vers un auteur parmi les premiers à recommencer à soupçonner que l'histoire des peuples barbares pouvait bien abriter en leurs «mystères» certaines nations mères encore méconnues des sciences et de la connaissance, c'est alors bien, par le truchement de quelque «archéologie», vers quelque livre sachant prendre en vue la "barbarie», «livre encore à faire - conclut-il » ${ }^{58}$, qu'il choisit pour sa part de faire signe.

\section{Épilogue. L'uniforme du général Cluseret}

Une profession de foi accompagne avec régularité les écrits dispersés par MelvilBloncourt dans la presse de nombreux pays ${ }^{59}$. C'est celle qui annonce la venue d'un jour de lumière que l'avenir garderait en réserve pour l'Afrique et qui pour Melvil-Bloncourt possède sa formule définitive dans des paroles prêtées par Voltaire à Mahomet: «le temps de l'Arabie est à la fin venu », auxquelles il suffirait d'ajouter: "Celui du Congo viendra aussi... L'humanité est patiente parce qu'elle est éternelle ${ }^{60}$. De cette promesse, Voltaire sera à tel point un emblème pérenne pour Melvil-Bloncourt que, tout en en espérant quelque argent pour subvenir à ses besoins «comme les naufragés de La Méduse attendaient une voile à l'horizon " ${ }^{61}$, il consacrera jusqu'à l'épuisement de ses forces son temps d'exil à Genève parmi d'autres communards à réaliser un écrit sur Voltaire si épais qu'il sera finalement publié en six volumes. Il n'en attendait bien sûr pas seulement le subside. Il fallait à Genève reconstituer d'une manière ou d'une autre le cénacle du café Voltaire de ses premières années à Paris, c'est-à-dire travailler à la réunion autour de livres, d'études, de publications et de projets d'écritures recommencés, ceux que les événements avaient éloignés de l'autre côté de la frontière. Et peut-être anticipait-il encore autre chose. En 1864, il avait fait paraître dans la Revue du Monde Colonial un texte inhabituel et prophétique dans lequel il décrivait un visiteur qui, parcourant les rues de Paris et découvrant les noms qu'elles portent, non seulement se serait donné le plaisir de voir ainsi les siècles écoulés de l'histoire de France se lever sous ses pas, mais aurait eu aussi à s'étonner de ne pas rencontrer des noms de rues

tatoués comme étant moins antihumains, moins cocasses, moins rares » que tels héros des romans de Sainte-Beuve (les Messieurs de Port-Royal). Voir G. Flaubert, Euvres complètes, t. II, Paris, Seuil, 1964, lettre à Sainte-Beuve du 23-24 décembre 1862, p. 751-754. Sur cette discussion et ses enjeux politiques et littéraires, se reporter par exemple à C. Saminadayar-Perrin, "Animalité, barbarie, civilisation: questions de frontière dans Salammbô", Revue Flaubert, $\mathrm{n}^{\circ}$ 10, 2010, ainsi que "Salammbô et la querelle du "roman archéologique" ", Revue d'histoire littéraire de la France, vol. III, 2011, p. 605-620.

58. S. Melvil-Bloncourt, «Critique littéraire», p. 76.

59. Parmi d'autres: S. Melvil-Bloncourt, «Critique littéraire», p. 373.

60. S. Melvil-Bloncourt, recension de Homme ou singe..., p. 147.

61. Lettre de S. Melvil-Bloncourt à Nadar du 31 janvier 1878, in Collection d'autographes formée par Felix et Paul Nadar, BNF, NAF 24278, XIX Megnin-Mols. 
présentifiant le Monde Colonial: rue « de la Guadeloupe, de la Martinique, de SaintDomingue, du Canada, de l'Acadie, de la Louisiane, de la Guyane, de Saint-Pierre et Miquelon, du Sénégal, de Gorée ${ }^{62}$, etc., ni non plus d'hommes illustres de ce même monde pour lesquels Melvil-Bloncourt suggère aussitôt une première liste de dix-sept noms. Vision anticipée, rusée et malicieuse d'un Paris à l'image d'une histoire réécrite de la France qui aurait su se couvrir d'autres monuments que ceux de son histoire étroitement nationale. C'est alors peut-être l'un de ces monuments entrevus en rêve que Melvil-Bloncourt aura choisi de rejoindre sous la Commune le jour où il alla offrir ses services au général Cluseret, chef militaire de la Commune et délégué à la guerre entre le 6 et le 30 avril 1871, à savoir cet homme qui, pour avoir été quelque temps général de l'armée nordiste durant la guerre de Sécession, s'obstina à traverser les événements parisiens en continuant de porter l'uniforme américain qui témoignait de, et pour, cet autre combat.

Stéphane DouaILLER Laboratoire d'études et de recherches sur les logiques contemporaines de la philosophie

Université Paris 8

62. S. Melvil-Bloncourt, "L'édilité parisienne et les colonies françaises», Revue du Monde Colonial, $3^{\mathrm{e}}$ série, t. XII, juillet 1864, p. 234. 